\title{
Editorial
}

Nephrology

Published online: June 29, 2016

\section{Resistance Exercise in the Hemodialysis Population - Who Should Do the Heavy Lifting?}

\author{
Kirsten L. Johansen \\ Division of Nephrology, University of California, San Francisco, Calif., USA
}

There have been numerous studies of exercise interventions to address the poor functional status of patients on dialysis [1]. However, most of these studies have been about aerobic exercise interventions, alone or in combination with another type of exercise or therapy, and resistance exercise (strength) training has been studied less frequently. Early studies of the physiology of debility in the dialysis population relied heavily on maximal exercise testing to measure peak oxygen consumption as an indicator of exercise capacity. Aerobic exercise training to boost the remarkably low exercise capacity observed in these studies was a logical choice. However, patients on dialysis also experience muscle atrophy and weakness [2], and protein energy wasting is associated with higher mortality [3]. These observations led some researchers to consider that interventions aiming to increase muscle strength or size might also improve physical functioning or other outcomes among patients on dialysis.

In this issue, Chan and Cheema [4] have reviewed the literature on resistance training in end-stage renal disease. Search of the literature yielded a total of 16 trials of resistance exercise training published from 2002 to 2014, ranging in size from 10 to 79 patients enrolled. In total, 299 patients were included in exercise groups, and fewer patients actually completed the exercise training inter-

\section{KARGER}

E-Mail karger@karger.com

www.karger.com/ajn ventions. Although outcomes differed widely across studies, the majority evaluated changes in muscle size and/or strength and most showed improvements. Most of the studies that assessed patients' self-reported physical functioning or their ability to stand repeatedly from a chair, a task that requires lower extremity strength but also coordination and balance, also demonstrated improvements. Other outcomes were less commonly evaluated and/or less consistently found to improve with exercise.

Although these results are encouraging in aggregate, not all of the news is good. The authors could not perform meta-analysis despite many studies measuring the same outcomes, a function of the wide variety of the interventions in terms of frequency, intensity, duration, and delivery. Most studies prescribed exercise during dialysis sessions, which in most cases meant limiting the exercise to lower extremities and using weighted ankle cuffs or elastic bands or tubes as the main source of resistance. Others used weight machines in an exercise facility (or in one case, in the dialysis facility prior to the dialysis session). The duration of exercise varied from 8 weeks to 6 months. Thus, although one can say that strength training appears to consistently increase muscle size and strength, studies have not clarified the most effective regimen or even the minimum load necessary to achieve benefit.
Kirsten L. Johansen, MD

Division of Nephrology, University of California, San Francisco VA Medical Center Nephrology Section, 111J, 4150 Clement Street

San Francisco, CA 94121 (USA)

E-Mail Kirsten.johansen@ ucsf.edu 
Outcomes varied widely as well. Although most studies evaluated changes in muscle size or strength in some way, the specific measures varied, and, surprisingly, 5 studies did not measure muscle strength, of which 4 also did not assess whether exercise increased muscle size. Lack of ascertainment of these key outcomes hampers interpretation of other results, as negative findings could reflect insufficient muscle overload, and positive results could be chance findings. Chan and Cheema [4] also highlighted the need for sufficiently detailed description of the intervention so that readers can evaluate the intensity of the intervention.

Several of the studies were not adequately controlled. Four studies did not include control groups, and another 2 did not include non-exercising control groups. Thus, it was not possible to compare changes in physical functioning between exercising and non-exercising patients for 137 (46\%) of the patients assigned to receive resistance exercise. This is important because improvements in tests of strength or other aspects of physical performance can occur as a result of familiarization with the task or equipment. Without a control group, such results might be interpreted as a benefit of exercise. The table in the Chen review provides a potential example of this phenomenon in the study by Segura-Ortí et al. [5], in which the exercise group experienced statistically significant improvement in several outcome measures compared to their own baseline performance, but these changes were not significantly different from changes in the control group. However, change in knee extension strength did differ significantly between groups, suggesting that there was a training-specific effect that may not have affected other measures of performance [5]. On the other hand, several studies have shown declines among non-exercising controls [6], suggesting that even maintenance of strength and function could be a benefit of exercise. Control groups are essential to avoid either a false positive result due to practice and familiarization or a false negative if intervention prevents clinically relevant decline. Although this may seem obvious, it is noteworthy that 3 of the 4 uncontrolled trials were among the more recently published studies.

The small sample sizes, variable study interventions, and problematic adherence are probably a reflection of the difficulty of implementing resistance exercise training among patients on dialysis rather than a lack of appreciation of proper study design. The high prevalence of frailty and debility makes the potential benefits of resistance exercise appealing but also makes implementation in the dialysis facility challenging. Small study sample sizes likely reflect the challenge of finding patients who are willing and able to participate. In addition, study dropout rates are often substantial, a phenomenon not highlighted by Chen et al., who extracted the number of patients assigned to exercise from the studies included in their review rather than the number of patients completing the programs and included in analyses.

Beyond the potentially compromising power and interpretation of study results, the high patient dropout is an indicator of the complexity of implementing strength training programs in patients on dialysis. In particular, the frequency of inter-current illness is high, with a median hospitalization rate that approaches 2 events per year in the United States [7]. Patients 'lose ground' during hospitalization, and exercise is often stopped. If exercise is to be resumed, patients' strength must be reevaluated and the exercise program recalibrated. Because of the importance of tailoring resistance exercise to be rigorous but safe for each patient, most or all of the published studies have been led by or have included exercise or health/fitness professionals. Likewise, resistance exercise training programs cannot be implemented in dialysis facilities outside of research studies without the support of these professionals.

Chen et al. conclude by calling for more high-quality research to delineate the benefits of resistance exercise among patients on dialysis, and it is hard to argue with this conclusion, given that the aforementioned problems make the current data far from definitive and do not allow us to identify a 'most effective' resistance exercise program for the dialysis population. However, it is not clear that the implied recommendation that resistance exercise be carried out in the dialysis facility (via trials testing exercise equipment 'customized to the dialysis setting') is the logical next step, at least in the United States, where there is no infrastructure or expertise available to implement or sustain such programs in dialysis facilities. The existing literature provides solid evidence that resistance exercise (at sufficient intensity and frequency) can lead to muscle hypertrophy and training-specific increases in strength among patients on dialysis; 'proof of principle' has been established. In order to move towards broader implementation, it may be more fruitful to make use of existing Medicare-supported exercise physiology or physical therapy services, letting these exercise professionals do the heavy lifting needed to develop customized exercise training regimens for our debilitated patients than to expect nephrologists and dialysis caregivers and providers to develop new skills and infrastructure. But that does not mean that nephrologists and dialysis caregivers should not be actively involved. We need to routinely assess the
Johansen 
strength and physical function in patients on dialysis, either by asking them or by administering simple tests, and to document the results. We should establish partnerships with exercise professionals so that they are familiar with our vulnerable patients. In addition, patients are more likely to participate in rehabilitation activities if such activities are discussed as part of the treatment plan when we see patients in the dialysis facility.

\section{Disclosure Statement}

Dr. K.L. Johansen is a Deputy Editor of the Clinical Journal of the American Society of Nephrology, is a member of the Steering Committee for the GSK Prolyl Hydroxylase Inhibitor study program, and receives research support from the National Institutes of Diabetes and Digestive and Kidney Diseases, the Centers for Disease Control and Prevention, and the California Department of Insurance. These activities do not present a conflict of interest with the content of this editorial.

\section{References}

1 Johansen KL: Exercise in the end-stage renal disease population. J Am Soc Nephrol 2007; 18:1845-1854.

2 Johansen KL, Shubert T, Doyle J, Soher B, Sakkas GK, Kent-Braun JA: Muscle atrophy in patients receiving hemodialysis: effects on muscle strength, muscle quality, and physical function. Kidney Int 2003;63:291297.

3 Fouque D, Kalantar-Zadeh K, Kopple J, Cano N, Chauveau P, Cuppari L, Franch H, Guarnieri G, Ikizler TA, Kaysen G, Lind- holm B, Massy Z, Mitch W, Pineda E, Stenvinkel $\mathrm{P}$, Treviño-Becerra $\mathrm{A}$, Wanner C: A proposed nomenclature and diagnostic criteria for protein-energy wasting in acute and chronic kidney disease. Kidney Int 2008;73: 391-398.

4 Chan D, Cheema B: Progressive resistance training in end stage renal disease: systematic review. Am J Nephrol 2016;44:32-45.

5 Segura-Ortí E, Kouidi E, Lisón JF: Effect of resistance exercise during hemodialysis on physical function and quality of life: random- ized controlled trial. Clin Nephrol 2009;71: 527-537.

6 Painter P, Carlson L, Carey S, Paul SM, Myll $\mathrm{J}$ : Physical functioning and health-related quality-of-life changes with exercise training in hemodialysis patients. Am J Kidney Dis 2000;35:482-492.

7 US Renal Data System: 2015 USRDS Annual Data Report: Epidemiology of Kidney Disease in the United States. Bethesda, National Institutes of Health, National Institute of Diabetes and Digestive and Kidney Diseases, 2015. 